\title{
Dolichopodidae (Diptera) fauna of the Novgorod Region. Addition
}

\section{Фауна Dolichopodidae (Diptera) Новгородской обцасти. Аополнение}

\author{
E.I. Ovsyannikova, I.Ya. Grichanov \\ Е.И. Овсянникова, И.Я. Гричанов
}

All-Russian Institute of Plant Protection, Podbelskiy roadway 3, St. Petersburg-Pushkin, 196608 Russia. E-mail: grichanov@mail.ru. Всероссийский институт защиты растений, шоссе Подбельского, 3, Санкт-Петербург-Пушкин, 196608, Россия.

KEY WORDS: Dolichopodidae, Russia, Novgorod Region, new records.

КЛЮЧЕВЫЕ СЛОВА: Dolichopodidae, Россия, Новгородская область, новые указания.

ABSTRACT. A new material of Dolichopodidae has been recently collected and identified, and includes 56 species found for the first time in the West of the Novgorod Region and 35 species new for the Region. In total, 20 genera and 89 species are recorded here that apparently makes up $40-50 \%$ of actual Dolichopodidae fauna in the Novgorod Region. This paper provides also distribution pattern for each collected species.

РЕЗЮМЕ. Приведены сведения о находках 56 видов семейства Dolichopodidae Новгородской области, в том числе 35 видов, новых для неё. Всего в области отмечено 20 родов и 89 видов, что, повидимому, составляет 40-50\% новгородской фауны Dolichopodidae. В статье указано также общее распространение для каждого отловленного вида.

The data of the known dolichopodid fauna of the Novgorod Region (or Novgorod Oblast) were recently summarized by Ovsyannikova and Grichanov [2019] who listed 44 species belonging to 14 genera. Comparing this fauna with faunas of the Leningrad and Pskov Regions, we should note that the territory of Novgorod Region remains undercollected, with all species collected from central and eastern parts (Borovichskiy, Krestetskiy and Valdaiskiy districts, environs of Velikiy Novgorod; Fig. 1). The period or sites of collection were usually unfavorable for the long-legged flies [Stackelberg, 1918; Ovsyannikova, Grichanov 2019].

The material for this study was collected by the authors of this paper during short-term visits to the Batetskiy district of the Novgorod Region in 2020 during the mass flight of dolichopodid imagoes, with special attention to six optimal for the flies wet habitats along the Luga (Rusynya and Zapol'e villages) and Udraika (Tashino and Shchepy villages) river banks and shores of small lakes (at Borki village and Borkovskoe Lake) (Fig. 2). The Batetskiy district as a whole

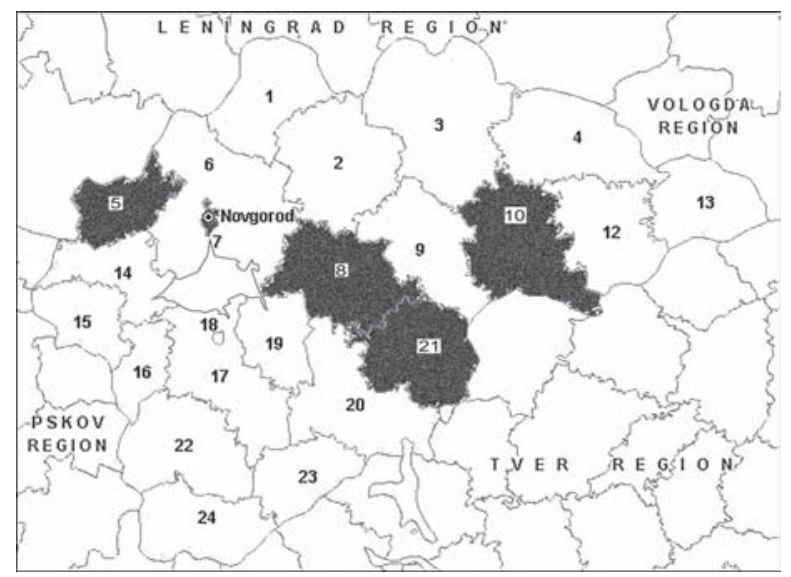

Fig. 1. Position of the Novgorod Region in the North-West of Russia and districts with known dolichopodid collections (marked with grey colour): 5 - Batetskiy district, 7 - Velikiy Novgorod, 8 Krestetskiy district, 10 - Borovichskiy district, 21 - Valdaiskiy district.

Рис. 1. Положение Новгородской области на северо-западе России и административных районов с известными сборами мух-зеленушек (с серой заливкой): 5 - Батецкий, 7 - Великий Новгород, 8 - Крестецкий, 10 - Боровичский, 21 - Валдайский.

is a lowland in the western part of the Region bordering with the Luzhskiy district of Leningrad Region and containing a number of marshes, rivulets and small lakes within mixed forests (Figs 3-6).

New records for 56 species are listed below, including 35 species new for the Novgorod Region, with entries arranged alphabetically. Collectors' names (the authors of this paper) are omitted in the list. The information on the global distribution for each species collected for the first time in the Novgorod Region follows Grichanov [2017]. For the complete distribution of other species see Ovsyannikova, Grichanov [2019]. The type localities are provided and the country lists are arranged alphabetical-

How to cite this article: Ovsyannikova E.I., Grichanov I.Ya. 2020. Dolichopodidae (Diptera) fauna of the Novgorod Region. Addition // Russian Entomol. J. Vol.29. No.3. P.307-314. doi: 10.15298/rusentj.29.3.12 


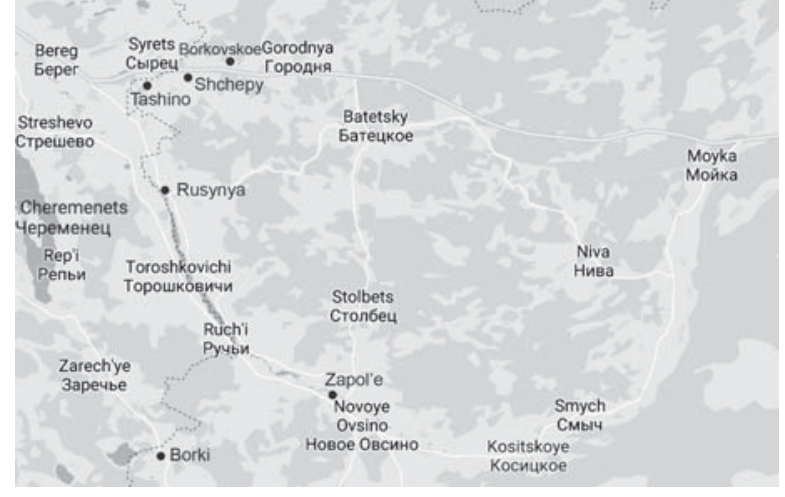

Fig. 2. Collection localities in the Batetskiy district (marked with circles).

Рис. 2. Места сбора в Батецком районе (отмечены точками).

ly. The words "Region" (oblast) and "Territory" (kray) are omitted from the list of Russian regions. Remarks are provided where deemed necessary. The collected material of the newly-recorded species has been mounted on
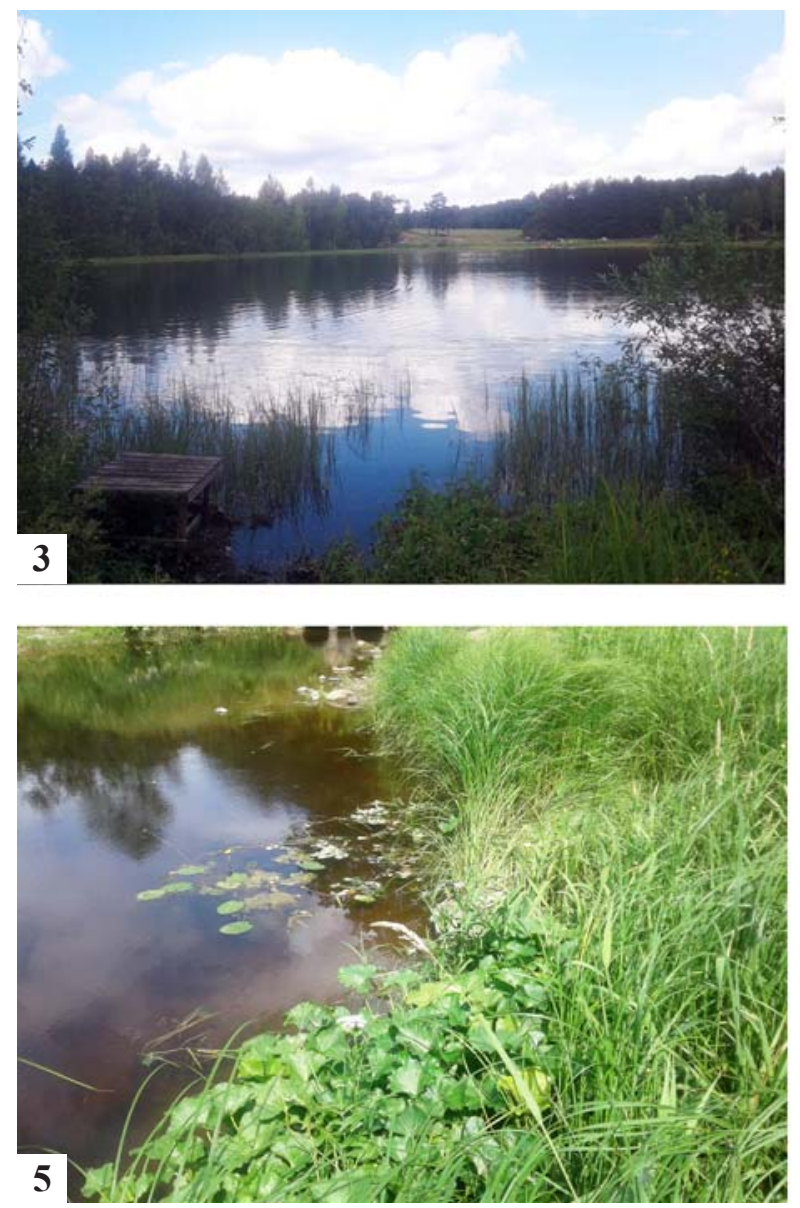

pins to be deposited at the Zoological Institute of the Russian Academy of Sciences.

\section{New Records}

Achalcus flavicollis (Meigen, 1824)

MATERIAL EXAMINED. Batetskiy dist., $10^{7}$, Zapolye, $58.509^{\circ} \mathrm{N}, 30.253^{\circ} \mathrm{W}, 29.06 .2020$.

DISTRIBUTION. Type locality: not given. Austria, Belgium, Bulgaria, Czech Republic, Denmark, Estonia, Finland, France, Germany, Ireland, Latvia, Lithuania, Netherlands, Norway, Poland, Romania, Russia (Leningrad, Pskov), Slovakia, Sweden, Switzerland, UK. New for Novgorod Region.

Argyra argentina (Meigen, 1824)

MATERIAL EXAMINED. Batetskiy dist., 1+, Tashino, $58.666^{\circ} \mathrm{N}, 30.101^{\circ} \mathrm{W}, 27.07 .2020$.

DISTRIBUTION. Type locality: not given [Europe]. Palaearctic: Austria, Azerbaijan, Belgium, Bosnia and Herzegovina, Croatia, Czech, Denmark, Estonia, Finland, France, Georgia, Germany, Greece, Hungary, Iran, Ireland, Latvia, Morocco, Netherlands, Norway, Poland, Romania, Russia (Adygea, Karachai-Cherkessia, Krasnodar, Leningrad, Pskov), Slovakia, Spain, Switzerland, Turkey, Ukraine, Sweden, UK. New for Novgorod Region.
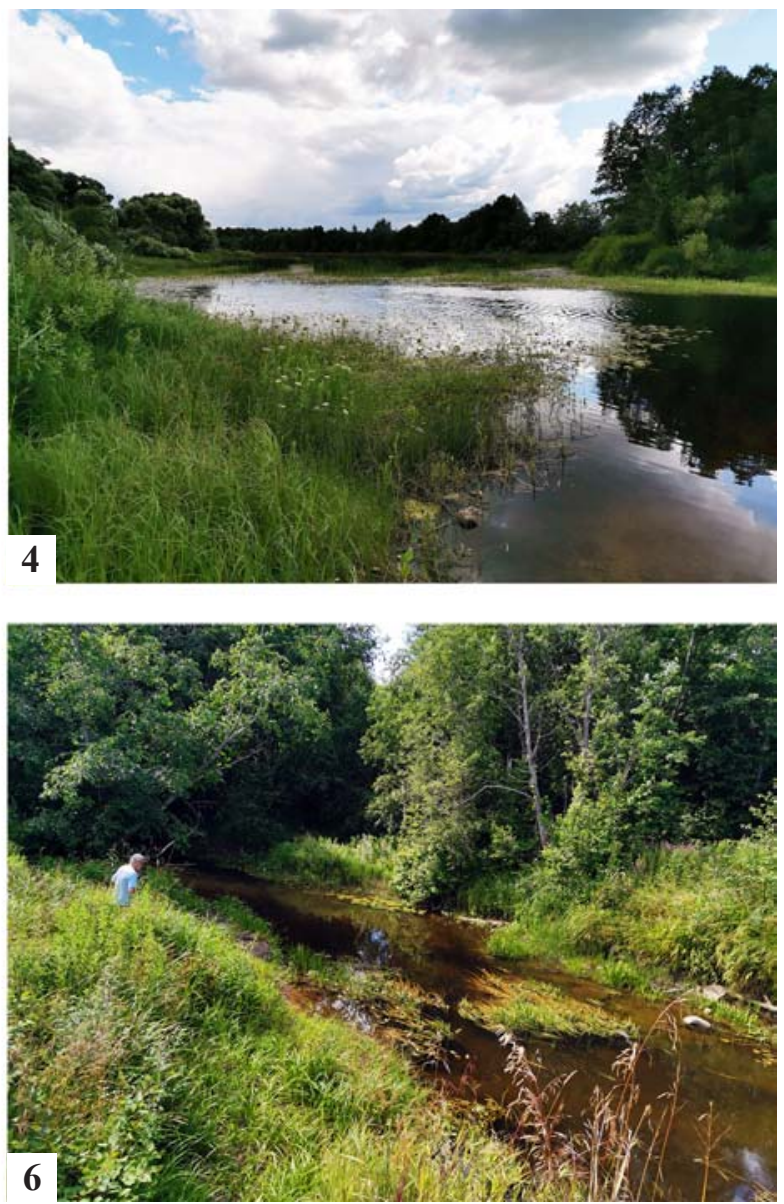

Figs 3-6. Collection localities: 3 - Borkovskoe Lake; 4 - Luga River near Rusynya; 5 - Udraika River bank near Shchepy; 6 - Udraika River near Tashino.

Рис. 3-6. Места сбора: 3 - озеро Борковское; 4 - река Луга под Русыней; 5 - берег реки Удрайка около Щепы; 6 -река Удрайка около Ташино. 
Argyra argyria (Meigen, 1824)

REFERENCES. Ovsyannikova, Grichanov 2019: 207 (Krestetskiy Dist., 24.08.2019).

MATERIAL EXAMINED. Batetskiy dist., $10^{\top}$, Shchepy, $58.670^{\circ} \mathrm{N}, 30.122^{\circ} \mathrm{W}, 13.07 .2020$.

DISTRIBUTION. Type locality: not given [probably Aachen, Germany]. Austria, Azerbaijan, Belarus, Belgium, Bulgaria, Croatia, Czech Republic, Denmark, Estonia, Finland, France, Germany, Greece (Crete), Hungary, Italy, Latvia, Moldova, Morocco, Netherlands, Norway, Poland, ?Romania, Russia (Crimea, Krasnodar, Leningrad, Lipetsk, Pskov, Vologda, Voronezh), Slovakia, Spain (incl. Canary Is.), Sweden, Switzerland, Turkey, UK, Ukraine.

\section{Argyra auricollis (Meigen, 1824)}

MATERIAL EXAMINED. Batetskiy dist., $1+$, Borki, $58.475^{\circ} \mathrm{N}$, $30.084^{\circ} \mathrm{W}, 29.06 .2020$.

DISTRIBUTION. Type locality: not given. Austria, Belgium, Bulgaria, Czech Republic, Denmark, Finland, France, Germany, Hungary, Italy, Latvia, Netherlands, Norway, Poland, Romania, Russia (Karelia, Leningrad), Slovakia, Sweden, Switzerland, UK. New for Novgorod Region.

Argyra diaphana (Fabricius, 1775)

MATERIAL EXAMINED. Batetskiy dist., $1+$, Borki, $58.475^{\circ} \mathrm{N}$, $30.084^{\circ} \mathrm{W}, 29.06 .2020$.

DISTRIBUTION. Type locality: Germany: Lipsiae [= Leipzig]. Austria, Azerbaijan, Georgia, Belarus, Belgium, Bosnia and Herzegovina, Bulgaria, Czech Republic, Denmark, Estonia, Finland, France, Georgia, Germany, Hungary, Iran, Ireland, Latvia, Moldova, Netherlands, Norway, Poland, Romania, Russia (Karelia, Krasnodar, Kursk, Krasnoyarsk, Leningrad, Lipetsk, Mordovia, Moscow, Pskov, Ryazan, Voronezh), Serbia, Slovakia, Sweden, Switzerland, Turkey, UK, Ukraine. New for Novgorod Region.

\section{Argyra elongata (Zetterstedt, 1843)}

MATERIAL EXAMINED. Batetskiy dist., $1^{\circ}$, Borki, $58.475^{\circ} \mathrm{N}$, $30.084^{\circ} \mathrm{W}, 29.06 .2020 ; 20^{7}$, Shchepy, $58.670^{\circ} \mathrm{N}, 30.122^{\circ} \mathrm{W}$, 13.07.2020.

DISTRIBUTION. Type locality: Sweden: "Ostrogothia ad Sorbyholm et Haradshammar". Belgium, Czech Republic, Denmark, Finland, France, Germany, Hungary, Ireland, Latvia, Netherlands, Norway, Poland, Russia (Leningrad, Voronezh), Sweden, Switzerland, UK, Ukraine (Odessa). New for Novgorod Region.

\section{Argyra vestita (Wiedemann, 1817)}

MATERIAL EXAMINED. Batetskiy dist., 2q, Rusynya, $58.608^{\circ} \mathrm{N}, 30.098^{\circ} \mathrm{W}, 13.07 .2020$.

DISTRIBUTION. Type locality: Germany: "bei Kiel". Austria, Belarus, Belgium, Bosnia and Herzegovina, Bulgaria, Czech Republic, Denmark, Estonia, Finland, France, Germany, Hungary, Iran, Ireland, Israel, Italy, North Macedonia, Netherlands, Norway, Poland, Romania, Russia (Krasnodar, Moscow, Novosibirsk, Ryazan, Vologda), Slovakia, Sweden, Switzerland, Turkey, UK. New for Novgorod Region.

\section{Campsicnemus armatus (Zetterstedt, 1849)}

MATERIAL EXAMINED. Batetskiy dist., $10^{7}$, Zapolye, $58.509^{\circ} \mathrm{N}, 30.253^{\circ} \mathrm{W}, 29.06 .2020$.

DISTRIBUTION. Type locality: Denmark: Rosenthal, Gryphium. Austria, Belgium, Czech Republic, Denmark, Finland, France, Germany, Iceland, Ireland, Italy, Mongolia, Netherlands, Norway, Poland, Russia (Arkhangelsk, Kamchatka, Karelia, Krasnoyarsk, Murmansk, Nenetsia, Tatarstan, Yakutia, Yamal, Yekaterinburg), Slovakia, Sweden, Turkey, UK. New for Novgorod Region.
Campsicnemus curvipes (Fallén, 1823)

REFERENCES. Ovsyannikova, Grichanov 2019: 207 (Krestetskiy Dist., 24.08.2019).

MATERIAL EXAMINED. Batetskiy dist., 1옹, Borki, $58.475^{\circ} \mathrm{N}, 30.084^{\circ} \mathrm{W}, 29.06 .2020 ; 70^{7}$, , Shchepy, $58.670^{\circ} \mathrm{N}$, $30.122^{\circ} \mathrm{W}, 13.07 .2020 ; 7 \sigma^{\top}, 1$, , Tashino, $58.666^{\circ} \mathrm{N}, 30.101^{\circ} \mathrm{W}$, 27.07.2020.

DISTRIBUTION. Type locality: not given [Sweden]. Abkhazia, Algeria, Armenia, Austria, Azerbaijan, Belarus, Belgium, Bulgaria, Czech Republic, Croatia, Denmark, Estonia, Finland, France, Georgia, Germany, Greece incl. Crete, Hungary, Iran, Ireland, Italy, Latvia, Luxembourg, Morocco, Netherlands, Norway, Poland, Portugal (Azores and Madeira), Romania, Russia(Adygea, Alania, Altai Terr., Belgorod, Chechnya, Crimea, Dagestan, Ivanovo, Kabardino-Balkaria, Kaluga, Karachay-Cherkessia, Karelia, Krasnodar, Krasnoyarsk, Kursk, Lipetsk, Leningrad, Moscow, Novosibirsk, Perm, Pskov, Ryazan, Stavropol, Tatarstan, Vologda, Voronezh), Serbia, Slovakia, Spain (incl. Canary Is.), Sweden, Switzerland, Turkey (Antalya, Bolu), UK, Ukraine.

\section{Campsicnemus pusillus (Meigen, 1824)}

MATERIAL EXAMINED. Batetskiy dist., 30', Shchepy, $58.670^{\circ} \mathrm{N}, 30.122^{\circ} \mathrm{W}, 13.07 .2020$.

DISTRIBUTION. Type locality: Germany: Hamburg. Austria, Belgium, Czech Republic, Estonia, Finland, France, Germany, Hungary, Ireland, Italy, Latvia, Norway, Poland, Romania, Russia (Bryansk, Ivanovo, Kabardino-Balkaria, Karelia, Leningrad, Pskov, Ryazan, Stavropol, Irkutsk, Kamchatka, Vladivostok), Slovakia, Sweden, UK. New for Novgorod Region.

\section{Campsicnemus scambus (Fallén, 1823)}

REFERENCES. Grichanov, 2012: 251 (Velikiy Novgorod env., 4.07.2012); Ovsyannikova, Grichanov 2019: 207 (Krestetskiy Dist., 24.08.2019).

MATERIAL EXAMINED. Batetskiy dist., $10^{7}$, Tashino, $58.666^{\circ} \mathrm{N}, 30.101^{\circ} \mathrm{W}, 27.07 .2020 ; 10^{\top}, 3$ \% , Borkovskoe Lake, $58.677^{\circ} \mathrm{N}, 30.161^{\circ} \mathrm{W}, 27.07 .2020$.

DISTRIBUTION. Type locality: Sweden: Esperod. Palaearctic: Austria, Belarus (Minsk), Belgium, Bulgaria, Czech Republic, Denmark, Estonia, Finland, France, Germany, Hungary, Ireland, Latvia, Lithuania, Netherlands, Norway, Poland, Romania, Russia (Altai Rep., Altai Terr., Arkhangelsk, Bashkortostan, Chelyabinsk, Yekaterinburg, Irkutsk, Khabarovsk, Khanty-Mansi, Kaliningrad, Kamchatka, Karelia, Komi, Krasnodar, Krasnoyarsk, Leningrad, Lipetsk, Mordovia, Moscow, Murmansk, Nenets, Novosibirsk, Pskov, Ryazan, Saratov, Tver, Tatarstan, Velikiy Novgorod, Vladivostok, Vologda, Voronezh, Yamalo-Nenets), Slovakia, Sweden, Switzerland, UK, Ukraine (Kherson, Odessa).

Chrysotus angulicornis Kowarz, 1874

MATERIAL EXAMINED. $1 \sigma^{7}, 1$ \% , Batetskiy dist., Rusynya, $58.608^{\circ} \mathrm{N}, 30.098^{\circ} \mathrm{W}, 13.07 .2020$.

DISTRIBUTION. Type locality: Insbrk [=Innsbruck, Austria]. Austria, Bulgaria, Czech Republic, Finland, France, Georgia, Iran, Italy, Lithuania, Poland, Romania, Russia (Alania, Dagestan, Karachai-Cherkessia, Krasnodar, Leningrad), Sweden, Switzerland, Turkey, Ukraine. New for Novgorod Region.

Chrysotus cilipes Meigen, 1824

REFERENCES. Stackelberg, 1918: 2153 (Borovichskiy dist., 25.07.1917).

MATERIAL EXAMINED. 20 ${ }^{\top}, 2 \circ$, Batetskiy dist., Shchepy, $58.670^{\circ} \mathrm{N}, 30.122^{\circ} \mathrm{W}, 13.07 .2020$.

DISTRIBUTION. Type locality: Germany: Hamburg. Trans-Palearctic species. Russia (Adygea, Altai Rep., Altai 
Terr., Astrakhan, Blagoveshchensk, Buryatia, Chita, Irkutsk, Kabardino-Balkaria, Karachai-Cherkessia, Karelia, Khabarovsk, Krasnodar, Krasnoyarsk, Moscow, Leningrad, Novgorod, Novosibirsk, Pskov, Rostov, Sakhalin, Tomsk, Vladivostok, Voronezh, Yakutia).

\section{Chrysotus femoratus Zetterstedt, 1843}

REFERENCES. Stackelberg, 1918: 2153 (Borovichskiy dist., 1-26.07.1917).

MATERIAL EXAMINED. $10^{7}$, Batetskiy dist., Zapolye, $58.509^{\circ} \mathrm{N}, 30.253^{\circ} \mathrm{W}, 29.06 .2020$.

DISTRIBUTION. Type locality: Denmark: Amager. Trans-Palearctic species. Russia (Adygea, Altai Rep., Arkhangelsk, Blagoveshchensk, Irkutsk, Kamchatka, Karelia, Karachay-Cherkessia, Khabarovsk, Krasnodar, Leningrad, Murmansk, Pskov, Ryazan, Sakhalin, Tatarstan, Velikiy Novgorod, Vladivostok, Voronezh, Yakutia).

\section{Chrysotus gramineus (Fallén, 1823)}

REFERENCES. Stackelberg, 1918: 2153 (Borovichskiy Dist., 30.06 - 30.07.1917); Ovsyannikova, Grichanov, 2019: 208 (Krestetskiy Dist., 24.08.2019).

MATERIAL EXAMINED. Batetskiy dist., $10^{7}, 3$, Zapolye, $58.509^{\circ} \mathrm{N}, 30.253^{\circ} \mathrm{W}, 29.06 .2020 ; 1$, Rusynya, $58.608^{\circ} \mathrm{N}$, $30.098^{\circ} \mathrm{W}, 13.07 .2020 ; 1$, , Borkovskoe Lake, $58.677^{\circ} \mathrm{N}, 30.161^{\circ} \mathrm{W}$, 27.07.2020

DISTRIBUTION. Type locality: not given [Sweden]. Trans-Palearctic species. Russia (Adygea, Alania, Altai Rep., Arkhangelsk, Bashkortostan, Blagoveshchensk, Buryatia, Chelyabinsk, Chita, Chukotka, Crimea, Irkutsk, KabardinoBalkaria, Karachay-Cherkessia, Karelia, Khabarovsk, Kostroma, Krasnodar, Krasnoyarsk, Kursk, Leningrad, Lipetsk, Mordovia, Moscow, Murmansk, Orel, Orenburg, Pskov, Ryazan, Sakhalin, Tatarstan, Tomsk, Velikiy Novgorod, Vologda, Voronezh, Yakutia, Yaroslavl).

\section{Chrysotus laesus (Wiedemann, 1817)}

MATERIAL EXAMINED. Batetskiy dist., 107, Rusynya, $58.608^{\circ} \mathrm{N}, 30.098^{\circ} \mathrm{W}, 13.07 .2020$.

DISTRIBUTION. Type locality: Germany: Kiel. TransPalearctic species. Russia (Adygea, Altai Rep., Bashkortostan, Blagoveshchensk, Buryatia, Chechnya, Chelyabinsk, Crimea, Dagestan, Ekaterinburg, Irkutsk, Karachai-Cherkessia, Karelia, Krasnodar, Krasnoyarsk, Kursk, Leningrad, Lipetsk, Magadan, Moscow, Orenburg, Omsk, Pskov, Ryazan, Tatarstan, Tomsk, Vladivostok, Voronezh, Yakutia, Yaroslavl). New for Novgorod Region.

Chrysotus neglectus (Wiedemann, 1817)

REFERENCES. Stackelberg, 1918: 2153 (Borovichskiy dist., 30.06 - 3.07.1917); Ovsyannikova, Grichanov, 2019: 208 (Valdaiskiy Dist., 1-3.08.1962).

MATERIAL EXAMINED. Batetskiy dist., 10 $0^{7}$, Rusynya, $58.608^{\circ} \mathrm{N}, 30.098^{\circ} \mathrm{W}, 13.07 .2020 ; 10^{7}, 1$ 웅, Borkovskoe Lake, $58.677^{\circ} \mathrm{N}, 30.161^{\circ} \mathrm{W}, 27.07 .2020$

DISTRIBUTION. Type locality: Germany: Holstein. Trans-Palearctic species. Russia (Alania, Altai, Arkhangelsk, Blagoveshchensk, Bryansk, Buryatia, Chechnya, Chelyabinsk, Chita, Dagestan, Kamchatka, Karelia, Khabarovsk, Krasnodar, Krasnoyarsk, Kursk, Leningrad, Mordovia, Moscow, Murmansk, Omsk, Pskov, Samara, Tomsk, Velikiy Novgorod, Vladivostok, Voronezh, Yakutia).

\section{Chrysotus suavis Loew, 1857}

MATERIAL EXAMINED. Batetskiy dist., 10 $0^{7}$, Shchepy, $58.670^{\circ} \mathrm{N}, 30.122^{\circ} \mathrm{W}, 13.07 .2020$.

DISTRIBUTION. Type locality: Germany: "Coln"; Austria: "Neusiedler See in Ungarn". Trans-Palearctic species.
Russia (Adygea, Alania, Altai Rep., Astrakhan, Blagoveshchensk, Bryansk, Buryatia, Irkutsk, Kabardino-Balkaria, Kamchatka, Khabarovsk, Krasnodar, Krasnoyarsk, Kursk, Leningrad, Lipetsk, Magadan, Novosibirsk, Pskov, Rostov, Ryazan, Sakhalin, Tatarstan, Voronezh, Yakutia). New for Novgorod Region.

\section{Diaphorus hoffmannseggi Meigen, 1830}

MATERIAL EXAMINED. Batetskiy dist., 10, 19 , Borki, $58.475^{\circ} \mathrm{N}, 30.084^{\circ} \mathrm{W}, 29.06 .2020$.

DISTRIBUTION. Type locality: not given. Austria, Belgium, Bosnia and Herzegovina, Bulgaria, Croatia, Czech Republic, Denmark, Finland, France, Germany, Hungary, Israel, Italy, Latvia, Netherlands, Poland, Romania, Russia (Leningrad, Moscow, North Caucasus, Ural, Voronezh), Serbia, Slovakia, Sweden, Switzerland, Turkey, UK. New for Novgorod Region.

Diaphorus oculatus (Fallén, 1823)

MATERIAL EXAMINED. Batetskiy dist., 10 $10^{\top}, 1$, Borki, $58.475^{\circ} \mathrm{N}, 30.084^{\circ} \mathrm{W}, 29.06 .2020$.

DISTRIBUTION. Type locality: Sweden: Westrogothia. Abkhazia, Austria, Belarus, Belgium, Bosnia and Herzegovina, Czech Republic, Denmark, Estonia, Finland, France, Germany, Hungary, Ireland, Italy, Latvia, Netherlands, Norway, Poland, Romania, Russia (Leningrad, Pskov, Ryazan), Slovakia, Sweden, Switzerland, UK, Ukraine. New for Novgorod Region.

\section{Dolichopus discifer Stannius, 1831}

REFERENCES. Stackelberg, 1918: 2153 (Borovichskiy dist., 30.06 - 5.07.1917)

MATERIAL EXAMINED. Batetskiy dist., $10^{7}$, Borki, $58.475^{\circ} \mathrm{N}$, $30.084^{\circ} \mathrm{W}, 29.06 .2020$.

DISTRIBUTION. Type locality: Germany. Holarctic species. Russia (Altai Rep., Arkhangelsk, Chukotka, Irkutsk, Karelia, Khabarovsk, Leningrad, Mordovia, Moscow, Murmansk, Sakhalin, Tatarstan, Velikiy Novgorod, Vladivostok, Vologda, Yakutia).

\section{Dolichopus longitarsis Stannius, 1831}

MATERIAL EXAMINED. Batetskiy dist., $10^{7}, 19$, Rusynya, $58.608^{\circ} \mathrm{N}, 30.098^{\circ} \mathrm{W}, 13.07 .2020 ; 1^{\circ}$, Borki, $58.475^{\circ} \mathrm{N}, 30.084^{\circ} \mathrm{W}$, 29.06 .2020

DISTRIBUTION. Type locality: Germany: Hamburg. Austria, Belarus, Belgium, Bosnia and Herzegovina, Bulgaria, ?Czech Republic, Denmark, Estonia, Finland, France, Georgia, Germany, Hungary, Iran, Ireland, Kazakhstan, Latvia, Lithuania, Netherlands, Norway, Poland, Romania, Russia (Altai, Karelia, Krasnoyarsk, Leningrad, Moscow, Nenetsia, Pskov, Saratov, Tatarstan, Vologda, Voronezh, Yakutia), Slovakia, Spain, Sweden, Switzerland, Turkey, UK, Ukraine. New for Novgorod Region.

Dolichopus pennatus Meigen, 1824

REFERENCES. Stackelberg, 1918, 2153 (Borovichskiy dist., 25.06 - 18.07.1917); Selivanova et al. 2019: 113 (Veliky Novgorod env., 22.06.1975).

MATERIAL EXAMINED. Batetskiy dist., 10 $0^{7}$, Zapolye, $58.509^{\circ} \mathrm{N}, 30.253^{\circ} \mathrm{W}, 29.06 .2020$.

DISTRIBUTION. Type locality: not given [Germany]. Trans-Palearctic species. Russia (Adygea, Alania, Altai Rep., Altai Terr., Arkhangelsk, Blagoveshchensk, Chechnya, Irkutsk, Kabardino-Balkaria, Kamchatka, Karachay-Cherkessia, Karelia, Khabarovsk, Krasnodar, Krasnoyarsk, Kuril Is., Leningrad, Magadan, Moscow, Murmansk, Pskov, Sakhalin, Sverdlovsk, Tatarstan, Velikiy Novgorod, Vladivostok, Vologda, Voronezh, Yakutia). 


\section{Dolichopus picipes Meigen, 1824}

MATERIAL EXAMINED. Batetskiy dist., 10, $10^{\circ}$, Borki, $58.475^{\circ} \mathrm{N}, 30.084^{\circ} \mathrm{W}, 29.06 .2020$

DISTRIBUTION. Type locality: not given. Austria, Belarus, Belgium, Bulgaria, Croatia, Czech Republic, Denmark, Estonia, Finland, France, Germany, Hungary, Ireland, Italy, Kazakhstan, Latvia, Netherlands, Norway, Poland, Romania, Russia (Altai Republic, Karelia, Krasnodar, Leningrad, Moscow, Murmansk, Pskov, Ryazan, Voronezh), Slovakia, Spain, Sweden, Switzerland, Turkey, UK. New for Novgorod Region.

Dolichopus plumipes (Scopoli, 1763)

REFERENCES. Stackelberg, 1918, 2153 (Borovichskiy dist., 26.06 - 30.07.1917); Ovsyannikova, Grichanov, 2019: 211 (Valdaiskiy Dist., 13.05.2018; Krestetskiy Dist., 24.08.2019).

MATERIAL EXAMINED. Batetskiy dist., $30^{2}, 5$, Zapolye, $58.509^{\circ} \mathrm{N}, 30.253^{\circ} \mathrm{W}, 29.06 .2020 ; 1$ 웅, Rusynya, $58.608^{\circ} \mathrm{N}$ $30.098^{\circ} \mathrm{W}, 13.07 .2020 ; 1$, , Shchepy, $58.670^{\circ} \mathrm{N}, 30.122^{\circ} \mathrm{W}$, 13.07.2020; 1 우, Borkovskoe Lake, $58.677^{\circ} \mathrm{N}, 30.161^{\circ} \mathrm{W}$, 27.07.2020

DISTRIBUTION. Type locality: Slovenia: "Carnioliae indigena". Mainly Holarctic species. N Russia (Arkhangelsk, Karelia, Komi, Leningrad, Murmansk, Nenets, Novgorod, Pskov, Vologda), C Russia (Belgorod, Kirov, Lipetsk, Mari El Republic, Moscow, Perm, Nizhniy Novgorod, Tatarstan, Voronezh), S Russia (Adygea, Alania, Kabardino-Balkaria, Karachay-Cherkessia, Krasnodar, Rostov), E Russia (Chukotka, Irkutsk, Kamchatka, Khabarovsk, Koryakia, Novosibirsk, Tomsk, Tyumen, Vladivostok); Neotropical: Mexico; Oriental: China, India (Kashmir).

\section{Dolichopus popularis Wiedemann, 1817}

MATERIAL EXAMINED. Batetskiy dist., $10^{7}, 29$, Zapolye, $58.509^{\circ} \mathrm{N}, 30.253^{\circ} \mathrm{W}, 29.06 .2020 ; 1^{\circ}$, Borki, $58.475^{\circ} \mathrm{N}, 30.084^{\circ} \mathrm{W}$, 29.06.2020.

DISTRIBUTION. Type locality: Germany: Holstein. Austria, Belarus, Belgium, Bulgaria, Bosnia and Herzegovina, Bulgaria, Czech Republic, Denmark, Estonia, Finland, France, Georgia, Germany, Hungary, Ireland, Netherlands, Norway, Poland, Romania, Russia (Altai Republic, Irkutsk, Krasnodar, Karachai-Cherkessia), Slovakia, Sweden, Switzerland, UK, Ukraine. New for Novgorod Region.

\section{Dolichopus simplex Meigen, 1824}

REFERENCES. Stackelberg, 1918, 2153 (Borovichskiy dist., 26.06 - 25.07.1917); Ovsyannikova, Grichanov, 2019: 211 (Krestetskiy Dist., 24.08.2019).

MATERIAL EXAMINED. Batetskiy dist., $20^{7}$, Rusynya, $58.608^{\circ} \mathrm{N}, 30.098^{\circ} \mathrm{W}, 13.07 .2020$.

DISTRIBUTION. Type locality: Germany: Hamburg, Kiel. Armenia, Austria, Belarus, Belgium, Czech Republic, Denmark, Estonia, Finland, France, Georgia, Germany, Hungary, Iran, Ireland, Kazakhstan, Latvia, Lithuania, Netherlands, Norway, Poland, Romania, Russia (Belgorod, Kaluga, Karachay-Cherkessia, Karelia, Kirov, Komi, Krasnodar, Kursk, Leningrad, Mordovia, Moscow, Murmansk, Nizhniy Novgorod, Orenburg, Pskov, Rostov, Ryazan, Saratov, Tatarstan, Novgorod, Vologda, Voronezh), Sweden, Switzerland, Turkey, UK, Ukraine.

\section{Dolichopus subpennatus d'Assis Fonseca, 1976}

REFERENCES. Ovsyannikova, Grichanov, 2019: 211 (Valdaiskiy Dist., 13.05.2018; Krestetskiy Dist., 24.08.2019).

MATERIAL EXAMINED. Batetskiy dist., $10^{7}$, Rusynya, $58.608^{\circ} \mathrm{N}, 30.098^{\circ} \mathrm{W}, 13.07 .2020 ; 20^{\top}, 3+$, Shchepy, $58.670^{\circ} \mathrm{N}$, $30.122^{\circ} \mathrm{W}, 13.07 .2020$.
DISTRIBUTION. Type locality: England: Invernessshire, Spey Bridge. Austria, Belgium, Czech Republic, Denmark, Estonia, Finland, France, Germany, Iran, Ireland, Lithuania, Luxembourg, Moldova, Netherlands, Norway, Poland, Romania, Russia (Adygea, Altai Republic, Kursk, Leningrad, Lipetsk, Perm, Voronezh), Slovakia, Sweden, Turkey, UK, Ukraine. New for Novgorod Region.

Dolichopus ungulatus (Linnaeus, 1758)

REFERENCES. Stackelberg, 1918: 2153 (Borovichskiy dist., 25.06 - 10.07.1917)

MATERIAL EXAMINED. Batetskiy dist., 10 $10^{\top}, 2+$, Shchepy, $58.670^{\circ} \mathrm{N}, 30.122^{\circ} \mathrm{W}, 13.07 .2020$.

DISTRIBUTION. Type locality: "Europe". Trans-Palearctic species. Russia (Adygea, Alania, Altai Republic, Altai Territory, Chechnya, Irkutsk, Kabardino-Balkaria, Karachay-Cherkessia, Karelia, Khabarovsk, Khanty-Mansi, Krasnodar, Krasnoyarsk, Kursk, Leningrad, Lipetsk, Mordovia, Moscow, Novosibirsk, Orenburg, Perm, Pskov, Ryazan, Tatarstan, Velikiy Novgorod, Vologda, Voronezh, Yakutia).

\section{Dolichopus wahlbergi Zetterstedt, 1843}

REFERENCES. Negrobov, 1979: 650 ("Novgorod Region" in a checklist; original publications have not been found).

MATERIAL EXAMINED. Batetskiy dist., 10 $0^{7}$, Rusynya, $58.608^{\circ} \mathrm{N}, 30.098^{\circ} \mathrm{W}, 13.07 .2020$.

DISTRIBUTION. Type locality: Sweden: "Ostrogothia ad Wadstena; Gottlandia, Stenkyrka". Austria, Belgium, Croatia, Czech Republic, Denmark, Estonia, Finland, France, Germany, Hungary, Ireland, N Kazakhstan, Netherlands, Norway, Poland, Romania, Russia (Karelia, Krasnodar, Leningrad, Murmansk, Stavropol, Novgorod, Voronezh, West Siberia, Yakutia), Slovakia, Spain, Sweden, Switzerland, UK. First reliable record from Novgorod Region.

Gymnopternus aerosus (Fallén, 1823)

REFERENCES. Stackelberg, 1918: 2153 (Borovichskiy dist., 30.06 - 22.07.1917); Ovsyannikova, Grichanov, 2019: 212 (Krestetskiy Dist., 24.08.2019)

MATERIAL EXAMINED. Batetskiy dist., 20 1 , 1, Borki, $58.475^{\circ} \mathrm{N}, 30.084^{\circ} \mathrm{W}, 29.06 .2020 ; 10^{7}, 4$ 우, Borkovskoe Lake, $58.677^{\circ} \mathrm{N}, 30.161^{\circ} \mathrm{W}, 27.07 .2020$.

DISTRIBUTION. Type locality: not given [Sweden]. Abkhazia, Austria, Belarus, Belgium, Bulgaria, Croatia, Czech Republic, Denmark, Estonia, Finland, France, Germany, Hungary, Ireland, Italy, Japan, Kazakhstan, Latvia, Lithuania, Mongolia, Netherlands, Norway, Poland, Romania, Russia (Adygea, Alania, Arkhangelsk, Buryatia, Irkutsk, Kaliningrad, Karelia, Karachay-Cherkessia, Khanty-Mansi, Krasnodar, Leningrad, Lipetsk, Mordovia, Moscow, Murmansk, Novgorod, Pskov, Tatarstan, "Ural", Voronezh, Vladivostok), Slovakia, Sweden, Tajikistan, UK, Ukraine; Oriental: Taiwan.

Gymnopternus brevicornis (Staeger, 1842)

REFERENCES. Ovsyannikova, Grichanov, 2019: 212 (Krestetskiy Dist., 24.08.2019).

MATERIAL EXAMINED. Batetskiy dist., 10 $10^{\top}$, Shchepy, $58.670^{\circ} \mathrm{N}, 30.122^{\circ} \mathrm{W}, 13.07 .2020 ; 10^{7}, 4$ ㅇ․ Borkovskoe Lake, $58.677^{\circ} \mathrm{N}, 30.161^{\circ} \mathrm{W}, 27.07 .2020$.

DISTRIBUTION. Type locality: not given [Denmark]. Austria, Belgium, Croatia, Czech Republic, Denmark, Finland, France, Germany, Hungary, Luxembourg, Netherlands, Norway, Poland, Romania, Russia (Altai Republic, Karelia, Khanty-Mansi, Leningrad, Murmansk, "Ural", Voronezh, Tatarstan, Vladivostok), Sweden, Switzerland, UK, Ukraine. New for Novgorod Region. 
Gymnopternus celer (Meigen, 1824)

REFERENCES. Stackelberg, 1918: 2153 (Borovichskiy dist., 3.07.1917).

MATERIAL EXAMINED. Batetskiy dist., $10^{7}, 2+$, Rusynya, $58.608^{\circ} \mathrm{N}, 30.098^{\circ} \mathrm{W}, 13.07 .2020$.

DISTRIBUTION. Type locality: not given [Germany]. Austria, Belgium, Bosnia and Herzegovina, Bulgaria, Croatia, Czech Republic, Denmark, Estonia, Finland, France, Germany, Hungary, Ireland, Italy, Karelia, Kazakhstan, Latvia, Lithuania, Luxembourg, Netherlands, Norway, Poland, Romania, Russia (Altai Republic, Altai Territory, Buryatia, Krasnodar, Krasnoyarsk, Mordovia, Moscow, Novosibirsk, Pskov, Ryazan, Tatarstan, Novgorod, Voronezh), Serbia, Slovakia, Sweden, Switzerland, Turkey, UK, Ukraine.

Gymnopternus metallicus (Stannius, 1831)

MATERIAL EXAMINED. Batetskiy dist., 707, 4, Borki, $58.475^{\circ} \mathrm{N}, 30.084^{\circ} \mathrm{W}, 29.06 .2020 ; 3{ }^{\circ}$, Borkovskoe Lake, $58.677^{\circ} \mathrm{N}$, $30.161^{\circ} \mathrm{W}, 27.07 .2020$.

DISTRIBUTION. Type locality: Germany: "Umgegend von Hamburg”. Abkhasia, Austria, Azerbaijan, Belarus, Belgium, Bulgaria, Czech Republic, Denmark, Estonia, Finland, France, Germany, Greece incl. Crete, Hungary, Iran, Ireland, Italy, Kazakhstan, Latvia, Lithuania, Moldova, Netherlands, North Macedonia, Norway, Poland, Romania, Russia (Alania, Altai Republic, Kabardino-Balkaria, Kamchatka, Karelia, KarachaiCherkessia, Khabarovsk, Krasnodar, Krasnoyarsk, Kursk, Leningrad, Moscow, Lipetsk, Orel, Pskov, Ryazan, Saratov, Tatarstan, Vladivostok, Vologda, Voronezh), Slovakia, Sweden, Switzerland, Turkey, UK. New for Novgorod Region.

Lamprochromus bifasciatus (Macquart, 1827)

MATERIAL EXAMINED. Batetskiy dist., $40^{7}$, Borki, $58.475^{\circ} \mathrm{N}$, $30.084^{\circ} \mathrm{W}, 29.06 .2020$.

DISTRIBUTION. Type locality: not given [North France]. Austria, Belgium, Bulgaria, Czech Republic, France, Germany, Hungary, Italy, Netherlands, Norway, Poland, Romania, Russia (Leningrad), Slovakia, Spain, Sweden, Switzerland, UK. New for Novgorod Region.

\section{Lamprochromus semiflavus (Strobl, 1880)}

MATERIAL EXAMINED. Batetskiy dist., 1으, Zapolye, $58.509^{\circ} \mathrm{N}, 30.253^{\circ} \mathrm{W}, 29.06 .2020$.

DISTRIBUTION. Type locality: Austria, Conventgarten. Austria, Belgium, Bulgaria, Czech Republic, Finland, France, Germany, Hungary, Netherlands, Poland, Portugal, Russia (Moscow, Orel, Pskov, Voronezh), Serbia, Turkey, UK. New for Novgorod Region.

\section{Rhaphium caliginosum Meigen, 1824}

MATERIAL EXAMINED. Batetskiy dist., 107, Rusynya, $58.608^{\circ} \mathrm{N}, 30.098^{\circ} \mathrm{W}, 13.07 .2020 ; 10^{7}$, Tashino, $58.666^{\circ} \mathrm{N}$, $30.101^{\circ} \mathrm{W}, 27.07 .2020$.

DISTRIBUTION. Type locality: not given. Algeria, $\mathrm{Ar}-$ menia, Austria, Azerbaijan, Bosnia and Herzegovina, Bulgaria, Denmark, Estonia, Finland, France, Germany, Greece, Israel and Golan Heights, Italy, Kazakhstan, Latvia, Morocco, Netherlands, North Macedonia, Norway, Romania, Russia (Adygea, Alania, Kabardino-Balkaria, Kaliningrad, Karachai-Cherkessia, Krasnodar, Krasnoyarsk, Kursk, Leningrad, Moscow, Murmansk, Pskov, Rostov, Stavropol, Tatarstan, Voronezh), Serbia, Sweden, Switzerland, Syria, Turkey, UK, Ukraine. New for Novgorod Region.

Rhaphium elegantulum (Meigen, 1824)

MATERIAL EXAMINED. Batetskiy dist., 19 , Borkovskoe Lake, $58.677^{\circ} \mathrm{N}, 30.161^{\circ} \mathrm{W}, 27.07 .2020$
DISTRIBUTION. Type locality: Germany: Hamburg. Palaearctic: Austria, Belarus, Belgium, Czech Republic, Denmark, Estonia, Finland, France, Germany, Ireland, Latvia, Moldova, Netherlands, Norway, Poland, Romania, Russia (Kamchatka, Karelia, Khantia-Mansia, Krasnoyarsk, Krasnodar, Leningrad, Murmansk, Novosibirsk, Voronezh, Baikal, "Polar Ural", "Igarka"), Spain, Sweden, UK; Nearctic Region. New for Novgorod Region.

\section{Rhaphium monotrichum Loew, 1850}

REFERENCES. Stackelberg, 1918: 2153 (as Xiphandrium monotrichum; Borovichskiy dist., 3.07.1917).

MATERIAL EXAMINED. Batetskiy dist., 10, 29 , Borki, $58.475^{\circ} \mathrm{N}, 30.084^{\circ} \mathrm{W}, 29.06 .2020 ; 1$ 웅, Zapolye, $58.509^{\circ} \mathrm{N}$, $30.253^{\circ} \mathrm{W}, 29.06 .2020$.

DISTRIBUTION. Type locality: Sweden: "Sueciam meridionalem et medium; in Scania ad Esperod, Ostrogothia ad Sudercopiam, ad Gusum, ad Walstena, ipsc. Etiam, Gottlandia, ad Holmiam". Austria, Belarus, Belgium, Bulgaria, Czech Republic, Denmark, Estonia, Finland, France, Germany, Hungary, Ireland, Italy, Netherlands, Norway, Poland, Romania, Russia ("Igarka", Irkutsk, Kabardino-Balkaria, Krasnoyarsk, Leningrad, Mordovia, Moscow, Murmansk, Novgorod, Voronezh), Slovakia, Sweden, Switzerland, UK.

Rhaphium aff. riparium (Meigen, 1824)

MATERIAL EXAMINED. Batetskiy dist., 1+, Shchepy, $58.670^{\circ} \mathrm{N}, 30.122^{\circ} \mathrm{W}, 13.07 .2020 ; 1$ 우 $\mathrm{T}$ ashino, $58.666^{\circ} \mathrm{N}$, $30.101^{\circ} \mathrm{W}, 27.07 .2020$

Notes. The females examined are most close to Rhaphium riparium (Meigen, 1824), which is known from the South of Leningrad Region of Russia and from many countries of Europe. It was reported and keyed as Porphyrops praerosa (Loew, 1850) in old literature.

Sciapus platypterus (Fabricius, 1805)

MATERIAL EXAMINED. Batetskiy dist., $20^{\top}, 1$, , Borki, $58.475^{\circ} \mathrm{N}, 30.084^{\circ} \mathrm{W}, 29.06 .2020$.

DISTRIBUTION. Type locality: Germany. Austria, Belarus, Belgium, Bosnia and Herzegovina, Bulgaria, Czech Republic, Denmark, Estonia, Finland, France, Germany, Greece, Hungary, Ireland, Italy, Latvia, Lithuania, Montenegro, Netherlands, Norway, Poland, Romania, Russia (Leningrad, Moscow, Pskov, Ryazan, Tatarstan, Voronezh), Serbia, Slovakia, Slovenia, Spain, Sweden, Switzerland, UK, Ukraine. New for Novgorod Region.

\section{Sybistroma obscurella (Fallén, 1823)}

MATERIAL EXAMINED. Batetskiy dist., $20^{7}, 1{ }^{\circ}$, Rusynya, $58.608^{\circ} \mathrm{N}, 30.098^{\circ} \mathrm{W}, 13.07 .2020$.

DISTRIBUTION. Type locality: Sweden: "Esperöd Scan". Abkhazia, Bosnia and Herzegovina, Bulgaria, Croatia, Czech Republic, Denmark, France, Georgia, Greece, Germany, Hungary, Ireland, Italy, Montenegro, ?Morocco, Netherlands, Romania, Russia (Adygea, Crimea, Krasnodar), Sweden, Switzerland, Turkey, UK. New for Novgorod Region.

Sympycnus aeneicoxa (Meigen, 1824)

REFERENCES. Ovsyannikova, Grichanov, 2019: 214 (Krestetskiy dist., 24.08.2019).

MATERIAL EXAMINED. Batetskiy dist., $80^{7}$, Borki, $58.475^{\circ} \mathrm{N}$, $30.084^{\circ} \mathrm{W}, 29.06 .2020 ; 40^{\circ}, 3$ ㅇ․ Borkovskoe Lake, $58.677^{\circ} \mathrm{N}$, $30.161^{\circ} \mathrm{W}, 27.07 .2020$

DISTRIBUTION. Type locality: not given [Germany]. Afghanistan, Austria, Belgium, Byelorussia, Czech Republic, Denmark, Estonia, Finland, France, Germany, Hungary, Italy, Latvia, Lithuania, Netherlands, Norway, Poland, Romania, Russia (Krasnoyarsk, Leningrad, Moscow, Novgorod, 
Pskov), Spain, Slovakia, Sweden, Switzerland, UK, Ukraine.

Sympycnus pulicarius (Fallén, 1823)

=Sympycnus annulipes (Meigen, 1824)

REFERENCES. Stackelberg, 1918: 2153 (as Sympycnus annulipes; Borovichskiy dist., 25.06.1917); Ovsyannikova, Grichanov, 2019: 214 (Krestetskiy Dist., 24.08.2019).

MATERIAL EXAMINED. Batetskiy dist., $150^{\top}$, 4우, Shchepy, $58.670^{\circ} \mathrm{N}, 30.122^{\circ} \mathrm{W}, 13.07 .2020$

DISTRIBUTION. Type locality: not given [Sweden]. Andorra, Austria, Azerbaijan, Belgium, Bosnia and Herzegovina, Bulgaria, Croatia, Czech Republic, Denmark, Estonia, Finland, France, Georgia, Germany, Greece, Hungary, Iran, Ireland, Italy, Kazakhstan, Kyrgyzstan, Latvia, Lithuania, Luxembourg, Macedonia, Moldova, Mongolia, Netherlands, Norway, Poland, Portugal, Romania, Russia (Alania, Altai Rep., Altai Ter., Buryatia, Chechnya, Chelyabinsk, Crimea, Kabardino-Balkaria, Kaliningrad, Karachay-Cherkessia, Karelia, Khakassia, Krasnodar, Krasnoyarsk, Leningrad, Lipetsk, Moscow, Murmansk, Novgorod, Novosibirsk, Pskov, Stavropol, Svedlovsk, Tambov, Tatarstan, Voronezh, Yakutia), Serbia, Slovakia, Spain incl. Canary Islands, Sweden, Switzerland, Tajikistan, Turkey, UK, Ukraine, Uzbekistan. Nearctic: USA (California).

Syntormon denticulatus (Zetterstedt, 1843)

MATERIAL EXAMINED. Batetskiy dist., 1\%, Rusynya, $58.608^{\circ} \mathrm{N}, 30.098^{\circ} \mathrm{W}, 13.07 .2020$.

DISTRIBUTION. Type locality: Sweden: Scania. Distribution: Abkhazia, Afghanistan, Armenia, Azerbaijan, Belorussia, Bosnia and Herzegovina, Bulgaria, Estonia, Finland, France, Germany, Greece, Israel, Italy, Kyrgyzstan, Norway, Poland, Romania, Russia (Adygea, Alania, Kabardino-Balkaria, Karelia, Leningrad, Moscow, Murmansk, Stavropol), Serbia, Sweden, Tajikistan, Turkey, UK, Ukraine, «North Africa». New for Novgorod Region.

\section{Syntormon monilis (Haliday, 1851)}

MATERIAL EXAMINED. Batetskiy dist., 107, Rusynya, $58.608^{\circ} \mathrm{N}, 30.098^{\circ} \mathrm{W}, 13.07 .2020$.

DISTRIBUTION. Type locality: England; Ireland. Distribution: Algeria, Austria, Belgium, Bulgaria, Czech Republic, Denmark, France, Germany, Hungary, Ireland, Italy, Latvia, Morocco, Netherlands, Poland, Romania; Russia (Kabardino-Balkaria, Krasnodar, Leningrad, Moscow, Ural), Serbia (?), Slovakia, Sweden, Switzerland, Tunisia, Turkey, UK. New for Novgorod Region.

Syntormon tarsatus (Fallén, 1823)

MATERIAL EXAMINED. Batetskiy dist., 107, 1, Borki, $58.475^{\circ} \mathrm{N}, 30.084^{\circ} \mathrm{W}, 29.06 .2020 ; 1$ ㅇ․ Z Zapolye, $58.509^{\circ} \mathrm{N}$, $30.253^{\circ} \mathrm{W}, 29.06 .2020 ; 1^{\circ}$, Borkovskoe Lake, $58.677^{\circ} \mathrm{N}, 30.161^{\circ} \mathrm{W}$, 27.07.2020.

DISTRIBUTION. Type locality: Sweden. Austria, Belarus, Belgium, Czech Republic, Denmark, Estonia, Finland, France, Germany, Hungary, Ireland, Latvia, Netherlands, Norway, Poland, Romania, Russia (Buryatia, Karelia, Kamchatka, Leningrad, Pskov), Slovakia, Sweden, UK, Ukraine (Kherson). New for Novgorod Region.

\section{Teuchophorus calcaratus (Macquart, 1827)}

MATERIAL EXAMINED. Batetskiy dist., 42 $0^{\top}$, Shchepy, $58.670^{\circ} \mathrm{N}, 30.122^{\circ} \mathrm{W}, 13.07 .2020 ; 5 \mathrm{O}^{\top}$, Tashino, $58.666^{\circ} \mathrm{N}$, $30.101^{\circ} \mathrm{W}, 27.07 .2020$.

DISTRIBUTION. Type locality: not given [France]. Austria, Azerbaijan, Belgium, Czech Republic, France, Germany, Georgia, Hungary, Italy, Latvia, Lithuania, Luxembourg, Netherlands, Poland, Romania, Russia (Adygea, Alania, Kab-
ardino-Balkaria, Krasnodar, Krasnoyarsk, Leningrad, Lipetsk, Mordovia, Moscow, Pskov, Vologda), Slovakia, Sweden, Switzerland, UK. New for Novgorod Region.

NOTES. Palaearctic Teuchophorus Loew, 1857 species are usually indistinguishable by females; therefore, most females collected are left unidentified.

Teuchophorus monacanthus Loew, 1859

MATERIAL EXAMINED. Batetskiy dist., $10^{7}$, Shchepy, $58.670^{\circ} \mathrm{N}, 30.122^{\circ} \mathrm{W}, 13.07 .2020$.

DISTRIBUTION. Type locality: not given. Austria, Azerbaijan, Belgium, Bulgaria, Croatia, Czech Republic, Denmark, France, Georgia, Germany, Greece incl. Crete, Hungary, Iran, Iraq, Ireland, Israel, Italy, Latvia, Luxembourg, Netherlands, North Macedonia, Norway, Poland, Romania, Russia (Adygea, Chechnya, Kabardino-Balkaria, Krasnodar, Leningrad, Lipetsk, Murmansk, Stavropol, Voronezh), Slovenia, Spain, Sweden, Switzerland, Turkey, UK, "Middle Asia". New for Novgorod Region.

Teuchophorus nigricosta (von Roser, 1840)

MATERIAL EXAMINED. Batetskiy dist., 39 $\sigma^{7}$, Shchepy, $58.670^{\circ} \mathrm{N}, 30.122^{\circ} \mathrm{W}, 13.07 .2020 ; 2 \sigma^{\top}, 1{ }^{\circ}$, Zapolye, $58.509^{\circ} \mathrm{N}$, $30.253^{\circ} \mathrm{W}, 29.06 .2020 ; 80^{\top}, 5^{\circ}$, Borki, $58.475^{\circ} \mathrm{N}, 30.084^{\circ} \mathrm{W}$, 29.06.2020; $20^{7}$, Tashino, $58.666^{\circ} \mathrm{N}, 30.101^{\circ} \mathrm{W}, 27.07 .2020$

DISTRIBUTION. Type locality: not given (Germany: Wurttemberg). Austria, Belgium, Czech Republic, Denmark, Estonia, Finland, France, Germany, Hungary, Italy, Netherlands, Poland, Romania, Russia (Krasnoyarsk, Leningrad, Lipetsk, Mordovia, Pskov), Slovakia, Sweden, Switzerland, UK. New for Novgorod Region.

Teuchophorus spinigerellus (Zetterstedt, 1843)

MATERIAL EXAMINED. Batetskiy dist., 10', Shchepy, $58.670^{\circ} \mathrm{N}, 30.122^{\circ} \mathrm{W}, 13.07 .2020 ; 130^{\gamma}$, Rusynya, $58.608^{\circ} \mathrm{N}$, $30.098^{\circ} \mathrm{W}, 13.07 .2020$; $2 \sigma^{\top}$, Tashino, $58.666^{\circ} \mathrm{N}, 30.101^{\circ} \mathrm{W}$, 27.07.2020.

DISTRIBUTION. Type locality: Suecia meridionali \& media, Scania ad Lund, Ostrogothia ad Wadstena, Dania [Sweden, Denmark]. Abkhazia, Austria, Azerbaijan, Belgium, Bulgaria, Croatia, Czech Republic, Denmark, Egypt; Estonia, Finland, France, Germany, Greece, Hungary, Ireland, Italy, S Kazakhstan, Latvia, Netherlands, North Macedonia, Norway, Poland, Romania, Russia (Adygea, Kabardino-Balkaria, Kaluga, Krasnodar, Leningrad, Pskov, Stavropol, Vologda), Spain, Sweden, Switzerland, Turkey, UK. New for Novgorod Region.

Thrypticus atomus Frey, 1915

MATERIAL EXAMINED. Batetskiy dist., 1옹 Shchepy, $58.670^{\circ} \mathrm{N}, 30.122^{\circ} \mathrm{W}, 13.07 .2020$.

DISTRIBUTION. Type locality: Finland: Karislojo. Austria, Belgium, Czech Republic, Finland, Hungary, Latvia, Netherlands, Russia (Arkhangelsk, Leningrad, Pskov, Ryazan, Yakutia, Krasnoyarsk), Sweden. New for Novgorod Region.

Thrypticus bellus Loew, 1869

MATERIAL EXAMINED. Batetskiy dist., 19, Zapolye, $58.509^{\circ} \mathrm{N}, 30.253^{\circ} \mathrm{W}, 29.06 .2020$.

DISTRIBUTION. Type locality: England: Kew. TransPalearctic and Afrotropical species. Russia (Krasnoyarsk, Leningrad, Novosibirsk, Rostov, Vladivostok, Voronezh). New for Novgorod Region.

Thrypticus intercedens Negrobov, 1967

MATERIAL EXAMINED. Batetskiy dist., 10', 1, Rusynya, $58.608^{\circ} \mathrm{N}, 30.098^{\circ} \mathrm{W}, 13.07 .2020$. 
DISTRIBUTION. Type locality: Russia: Borok, Jaroslavl region. Finland, France, Germany, Iran, Norway, Russia (Khantia-Mansia, Sayan Mnts., Yakutia, Yaroslavl), Sweden, Turkey. New for Novgorod Region.

\section{Discussion}

As a result of this study, new material of Dolichopodidae was collected and identified. The present research features new records, including 35 species found for the first time in the Novgorod Region. In total, 20 genera and 89 species are known from the Region. The latter number apparently makes up $40-50 \%$ of actual Dolichopodidae fauna in the Novgorod Region.

Most collected species are widespread across the Palaearctic Region, being common in the well-studied neighboring Regions of Russia. For example, the fauna of Leningrad Region numbers 223 dolichopodid species [Grichanov, Ovsyannikova, 2017] and that of Pskov Region includes 99 species collected mainly in the South of the Region [Grichanov, Ovsyannikova, 2015], i.e. about $250 \mathrm{~km}$ southward of the Batetskiy district of Novgorod Region. The Luzhskiy district of Leningrad Region bordering with the Batetskiy district is the best studied in the Region. For example, Stackelberg [1962] reported 104 dolichopodid species in the Luzhskiy district ( $51 \%$ of the then known Leningrad Region fauna) in addition to about 20 species "common all over the Region". However, 25 species in his list belonged to dendrophilous genera Medetera Fischer von Waldheim, 1819, Sciapus Zeller, 1842 and Systenus Loew, 1857, which we usually ignored in our recent field researches. All species listed in this paper were collected along river banks and lake shores on grass by sweep net (see Fig. 2).

Some rare dolichopodid species collected in the Batetskiy district are worth noting. Achalcus flavicollis is a boreal European species having at present the easternmost distribution in Russia (Leningrad, Novgorod and Pskov Regions). Argyra auricollis has similar distribution pattern, being reported in Russia from Leningrad, Novgorod Regions and Karelia only, as well as Diaphorus oculatus (Leningrad, Novgorod, Pskov and Ryazan Regions). The European Argyra elongata was found only in Leningrad, Novgorod and Voronezh Regions of Russia. Campsicnemus armatus is probably a northern representative in the Novgorod Region fauna, being at present unknown in Leningrad and Pskov Regions (a few records from southern Palearctic countries must be confirmed). In contrast, Sybistroma obscurella is a southern West Palearctic element with the nearest findings in Adygea, Crimea, Krasnodar Territory of Russia, Hungary and southern Sweden. Lamprochromus bifasciatus is rediscovered in Russia, about 90 years since it was last found in the Luzhskiy district of Leningrad Region.

Acknowledgements. The authors are sincerely grateful to Dr. Timur Yusupov (Luga, Leningrad Region, Russia), for the field trips to Novgorod Region localities. The work was performed within the Program for Basic Scientific Researches in the Russian Federation for the Long-Term Period (20132020) approved by Order No. 2538-r of the Government of the Russian Federation dated December 27, 2012, and supported by the All-Russian Institute of Plant Protection project No. 0665-2019-0014.

Competing imterests. The authors declare no competing interests.

\section{References}

Grichanov I.Ya. 2012. A new species of Campsicnemus from the Far East of Russia with some new records (Dolichopodidae, Diptera) // Amurian Zoological Journal. Vol.4. No.3. P.250-252.

Grichanov I.Ya. 2017. Alphabetic list of generic and specific names of predatory flies of the epifamily Dolichopodoidae (Diptera). $2^{\text {nd }}$ ed. Saint Petersburg: All-Russian Research Institute of Plant Protection. 563 p. DOI: 10.5281/zenodo. 884863.

Grichanov I.Ya., Ovsyannikova E.I. 2015. New records of Dolichopodidae from Pskov Region of Russia (Diptera: Empidoidea) // CESA News. No.110. P.1-5.

Grichanov I.Ya., Ovsyannikova E.I. 2017. First data on Dolichopodidae (Diptera) of the West Kotlin Nature Reserve (Saint Petersburg, Russia) // Acta Biologica Sibirica. Vol.3. No.2. P.52-57. DOI: $10.14258 /$ abs.v3i2.2732.

Negrobov O.P. 1979. [Family Dolichopodidae (Diptera) of the fauna of USSR. I. Subfamilies Dolichopodinae and Medeterinae] // Entomologicheskoe Obozrenie. Vol.58. No.3. P.646-659 [in Russian]

Ovsyannikova E.I., Grichanov I.Ya. 2019. New records of longlegged flies (Diptera, Dolichopodidae) from Novgorod Region of Russia with an annotated checklist of species // Amurian Zoological Journal. Vol.11. No.3. P.206-217. DOI: 10.33910/ 2686-9519-2019-11-3-206-217.

Selivanova O.V., Negrobov O.P., Maslova O.O. 2019. New data on the systematics and fauna of Dolichopus subpennatus D'Assis Fonseca, 1976 and Dolichopus pennatus Meigen, 1824 (Dolichopodidae, Diptera) // Acta Biologica Sibirica. Vol.5. No.2. P.111-114. DOI: 10.14258/ABS.V5.I2.6193.

Stackelberg A.A. 1918. [On the Diptera fauna of the Novgorod Province] // Izvestiya Rossiiskoi akademii nauk. VI seriya. Vol.12. No.18. P.2149-2160 [in Russian].

Stackelberg A.A. 1962. [Materials on Diptera of the Leningrad Region. V. Dolichopodidae] // Trudy Zoologicheskogo instituta AN SSSR. Vol.31. P.280-317 [in Russian]. 